\title{
LA TRANSFERENCIA DE LAS ESCUELAS PRIMARIAS NACIONALES A LAS PROVINCIAS DURANTE LA ÚLTIMA DICTADURA MILITAR EN ARGENTINA (ENTRE RÍOS, 1976-1978)
}

\author{
Eva Mara Petitti ${ }^{\star}$
}

\section{RESUMEN}

Este artículo aborda una problemática que ha representado una inflexión en el sistema educativo argentino: la transferencia de escuelas primarias nacionales a las provincias en el marco de la última dictadura militar. Poniendo el foco en el periodo 1976-1978 y profundizando en la provincia de Entre Ríos, este trabajo pretende evidenciar la oposición de las provincias a la transferencia, las diferencias entre las gestiones de los ministros de Educación Juan Catalán y Ricardo Bruera, la necesidad de legitimar la medida por parte del gobierno dictatorial y el proceso de adaptación administrativa de las provincias una vez concretada la medida.

Palabras clave: transferencia, escuelas primarias, dictadura militar, Entre Ríos.

\footnotetext{
* Instituto de Estudios Sociales - Universidad Nacional de Entre Ríos (UNER), Entre Rios, Argentina.
} 


\title{
A TRANSFERÊNCIA DAS ESCOLAS PRIMÁRIAS NACIONAIS PARA AS PROVÍNCIAS DURANTE A ÚLTIMA DITADURA MILITAR NA ARGENTINA (ENTRE RIOS, 1976-1978)
}

\begin{abstract}
RESUMO
Este artigo aborda um problema que tem representado um ponto de viragem no sistema educativo argentino: a transferência das escolas primárias nacionais para as províncias sob a última ditadura militar. Focalizando o período 1976-1978 e aprofundando na província de Entre Ríos, este documento procura destacar a oposição das províncias à transferência, as diferenças entre os esforços dos Ministros da Educação Juan Catalán e Ricardo Bruera, a necessidade de legitimar a medida pelo governo ditatorial e o processo de adaptação administrativa das províncias uma vez que a medida seja implementada.
\end{abstract}

Palavras-chave: transferência, escolas primárias, ditadura militar, Entre Ríos.

\section{THE TRANSFER OF NATIONAL PRIMARY SCHOOLS TO THE PROVINCES DURING THE LAST MILITARY DICTATORSHIP IN ARGENTINA (ENTRE RIOS, 1976-1978)}

\section{ABSTRACT}

This article addresses a problem that has represented a turning point in the Argentine education system: the transfer of national primary schools to the provinces under the last military dictatorship. Focusing on the period 1976-1978 and delving into the province of Entre Ríos, this paper seeks to highlight the opposition of the provinces to the transfer, the differences between the efforts of the Ministers of Education Juan Catalán and Ricardo Bruera, the need to legitimize the measure by the dictatorial government and the process of administrative adaptation of the provinces once the measure is implemented.

Keywords: transference, primary Schools - military dictatorship, Entre Ríos.

\section{LE TRANSFERT DES ÉCOLES PRIMAIRES NATIONALES AUX PROVINCES PENDANT LA DERNIÈRE DICTATURE MILITAIRE EN ARGENTINE (ENTRE RÍOS, 1976-1978)}

RÉSUMÉ

Cet article aborde un problème qui a représenté un tournant dans le système éducatif argentin: le transfert des écoles primaires nationales aux provinces sous la dernière dictature militaire. En se concentrant sur la période 1976-1978 et en se penchant sur la province d'Entre Ríos, ce document cherche à mettre en évidence l'opposition des provinces au transfert, les différences entre les 
efforts des ministres de l'Éducation Juan Catalán et Ricardo Bruera, la nécessité de légitimer la mesure par le gouvernement dictatorial et le processus d'adaptation administrative des provinces une fois la mesure mise en œuvre.

Mots-clés: transfert, écoles primaries, dictature militaire, Entre Ríos. 


\section{INTRODUCCIÓN}

En junio de 1978, en el marco de la más cruenta dictadura que vivió la región y de una fuerte crisis económica, un diario local expresaba que "se ha concretado masivamente el hecho del sistema educativo que históricamente exigió mayor cantidad de análisis, tramitaciones y decisiones de las más diversas índoles". ${ }^{1}$ Con ello refería a la promulgación de las leyes 21.809 y 21.810 de transferencia de servicios educativos que traspasaron alrededor de 6.500 escuelas pre primarias y primarias, supervisiones y juntas de clasificación que hasta entonces dependían del Consejo Nacional de Educación (CNE), a la Municipalidad de la Ciudad de Buenos Aires, el Territorio Nacional de la Tierra del Fuego, Antártida e Islas del Atlántico Sur (en adelante Tierra del Fuego) y a la mayor parte de las provincias². De esta forma, ocasionó grandes problemas a las jurisdicciones subnacionales que tuvieron que incorporar los establecimientos nacionales sin disponer de los recursos económicos necesarios para su sostenimiento y en algunos casos derivó en el cierre y la racionalización del personal (GUDELEVICIUS, 2013).

Por aplicación de los principios federales de la carta constitucional sancionada en 1853, el sostenimiento de la educación básica estaba a cargo de las provincias. Los establecimientos dependientes del CNE en los diferentes espacios subnacionales se crearon, por un lado, en el marco de la Ley de Educación Común 1420/884 que regía en la ciudad de Buenos Aires y los territorios nacionales que fueron provincializados en la década del '50 y, por otro lado, encuadrados en la Ley Láinez 4874/905.3

\footnotetext{
${ }^{1}$ El Diario, 7/6/78, p. 1.
}

2 A comienzos del periodo dictatorial, de las 22 provincias existentes en ese entonces, habían transferido sus escuelas primarias nacionales Santa Cruz, Río Negro, La Rioja y Buenos Aires.

3 Como señalan Ayrolo y Verdo, "la tensión entre las dos soberanías, la nacional y la provincial" no quedó resuelta con la primera experiencia de unidad política de la mayoría de las provincias sellada en 1853 (2016. p. 3). Así, la carta constitucional definió una forma de gobierno federal fundada "en la delegación voluntaria de sus atribuciones con el objetivo de constituir un entramado institucional central" (BRAVO, 2013). En su artículo 5 estableció que los gobiernos provinciales dictarían sus propias constituciones asegurando "su administración de justicia, su régimen municipal y la educación primaria gratuita”. Por el artículo 67, le correspondía al 
En los últimos años se han realizado numerosos avances en lo que respecta al conocimiento de las políticas educativas durante la última dictadura (GARCÍA, 2019). 4 Como ha sido mostrado en dichas investigaciones, uno de sus pilares fue la descentralización. Bajo dicho concepto, se articularon dos políticas diferentes aunque interconectadas. Por un lado, la regionalización del curriculum, como mecanismo de adecuación a las posibilidades de cada región de garantizar una injerencia mayor de las realidades locales. Por otro, la transferencia del servicio educativo a los espacios subnacionales. Esta última, encontró antecedentes en una normativa de 1956 y se inició en la década del '6o, si bien con una diferencia substancial respecto al carácter compulsivo que tuvo la efectuada en 1978 (GUDELEVICIUS, 2009).

Desde inicios del gobierno democrático la transferencia ha despertado el interés de los investigadores (TEDESCO; BRASLAVSKY; CARCIOFI, 1983; BRASLAVSKY, 1885; FILMUS, 1998). En el cambio de siglo, y a la luz de los resultados de la extensión de la descentralización en los niveles medio y terciario, los trabajos de Alonso Brá (1999), Falleti (2001) y Rivas (2004) la enmarcan en el problema más amplio de la relación a largo plazo entre las provincias y la nación argentina. En última década se ha avanzado en esta tendencia (RUIZ; MAUCERI, 2009; RODRÍGUEZ, 2009; BORDÓN 2010; MORDUCHOWICZ, 2009, 2010) y se ha profundizado en el periodo de la dictadura. A partir de los estudios sobre la provincia de Buenos Aires (RODRÍGUEZ, 2008a, 2008b) y la Municipalidad homónima (GUDELEVICIUS, 2013), sabemos que no tuvo un desarrollo unívoco en el tiempo ni entre las distintas jurisdicciones argentinas. Asimismo conocemos que si bien el contexto de terrorismo de estado posibilitó

Congreso de la Nación "acordar subsidios del Tesoro nacional a las provincias cuyas rentas no alcancen, según sus presupuestos, a cubrir gastos ordinarios" y "proveer lo conducente a la prosperidad del país, al adelanto y bienestar de todas las provincias..." Basada en este último, la ley 4874/905 impulsada por el senador Manuel Láinez, otorgó al CNE la facultad de establecer en aquellas provincias que lo solicitaran -salvando así la inconstitucionalidad que podía suponer esta medida- escuelas elementales (hasta cuarto grado), infantiles (hasta segundo), mixtas y rurales teniendo en cuenta el porcentaje de analfabetos.

4 Natalia García (2019) ofrece un interesante y actualizado estado de la cuestión sobre educación y dictadura. 
su concreción al limitar la capacidad de oposición social, el proceso no estuvo exento de conflictos y denuncias (RODRÍGUEZ, 2008a; 2008b; GUDELEVICIUS, 2009; 2013; DE LUCA, 2013).

Las categorías analíticas que orientaron la investigación empírica son las de descentralización y federalismo. Dora Orlansky, siguiendo a Remy Prud' homme, comparte la afirmación de que la ambigüedad del concepto descentralización contribuye a su atracción (ORLANSKY, 1994, p. 3). Su conjugación con el concepto de federalismo, cuya imprecisión se sustenta en la compleja y cambiante relación entre el poder central y las provincias construida sobre la base de la Constitución de 1853 (BRAVO, 2013), otorgó legitimidad a una medida llevada a cabo por la última dictadura militar y resistida por los gobiernos subnacionales.

No se trató de un asunto exclusivamente nacional. Como muestra Dora Orlansky "hacia el fin de la década de 1970 la descentralización obtuvo un amplio apoyo alrededor del mundo como la solución propuesta ante los problemas crónicos de eficiencia y equidad en el sector público" (1998, p. 16). La descentralización formó parte del discurso de los países centrales y fue respaldada por préstamos de los organismos internacionales que operaban en Latinoamérica (KRAWCZYK, 2002; RODRÍGUEZ; 2008b; ASCOLANI 2008). En un trabajo anterior hemos visto que la medida coincidió con la introducción de financiamiento externo orientado a la educación en las áreas rurales, que eran aquellas donde había tenido mayor presencia las escuelas dependientes del CNE en las provincias (PETITTI, 2019).

En este artículo analizaremos las tensiones administrativas y fiscales entre la nación y las provincias que se desplegaron en torno al proceso de transferencia que se llevó a cabo durante la última dictadura militar. Para ello nos centraremos en los tres primeros años (1976-1978) y tomaremos como unidad de análisis la provincia de Entre Ríos. Ésta, que no había participado de los convenios firmados durante la década del '6o, tenía un porcentaje de escuelas nacionales relativamente bajo en comparación con la mayor parte de provincias. 
Por otra parte, durante la gestión del ministro de Educación Juan Catalán, ocupó un lugar privilegiado en la delegación de la región del Noroeste Argentino (NEA). 5

Nos interesa señalar que las presiones de los gobiernos provinciales, de los supervisores nacionales y del CNE, implicaron, además de postergar la transferencia y retroceder en la intensión de traspasar el nivel medio, diferentes estrategias para buscar legitimidad. Por otra parte, pretendemos mostrar las acciones que debieron llevar a cabo las provincias para sortear las dificultades administrativas y fiscales que significaba hacerse cargo de las escuelas nacionales, así como advertir que la falta de datos exactos sobre el número de traspasos contribuyó a ocultar la magnitud de los cierres.

Para ello realizamos una investigación cualitativa basada en enfoques y métodos históricos de relevamiento de documentos y su correspondiente análisis heurístico e interpretación histórica. Trabajamos con un acervo documental en su mayoría inédito, localizado en el Archivo Histórico de la provincia de Entre Ríos y el archivo del Consejo General de Educación (CGE), con publicaciones que se encuentran en la Biblioteca Nacional de Maestros, así como también con artículos de prensa. Para su análisis se articularan los aportes teóricos de las investigaciones que tienen por objeto la observación del Estado "desde adentro" (BOHOSLAVSKY; SOPRANO, 2010) y el proceso de implementación de las políticas educativas durante la dictadura, reconociendo las disputas y negociaciones sobre sus objetivos y la búsqueda de legitimidad (RODRÍGUEZ, 2009).

\footnotetext{
${ }^{5}$ Las cinco regiones educativas creadas por el Consejo Federal de Educación son: Sur, Centro, Cuyo, Noroeste Argentino (NEA) y Noroeste Argentino (NOA). La región del NEA, además de Entre Ríos, la integraban Corrientes, Misiones, Formosa y Chaco. En 1930 un 33\% de la matrícula de todo el país asistía a escuelas nacionales. Esa proporción se elevaba a un 55\% en la región del NEA, con importantes variaciones en su interior. Mientras que en Entre Ríos representaban un 24\%, en Misiones y Chaco ocupaba más del 70\%.
} 


\section{LA GESTIÓN DE BRUERA: LA BÚSQUEDA DE UN ACUERDO PARA TRANSFERIR LAS ESCUELAS}

Poco después de iniciado el golpe de Estado y en el marco de una política económica de recorte presupuestario, volvió a funcionar el Consejo Federal de Educación (CFE) creado en 1972. En mayo de 1976 se reunió en la ciudad de Buenos Aires la III Asamblea Extraordinaria presidida por el ministro de Educación nacional, Ricardo Bruera, donde se acordó "intensificar una coordinación efectiva entre Nación y Provincias, a fin de posibilitar la descentralización educativa". ${ }^{6}$ Bruera, quien había ocupado ese cargo en la provincia de Santa Fe entre 1970 y 1973, afirmaba que "la desburocratización y la descentralización serán las dos bases fundamentales de la política educativa nacional".7 Avanzando un poco más sobre la temática, el vicepresidente del Comité Ejecutivo del CFE, ministro de Educación de Córdoba, expresaba que la "superposición de escuelas" era preocupante: "Ya no puede seguir habiendo feudos o que en un mismo territorio haya escuelas nacionales y provinciales sin ninguna coordinación entre sí". 8

Bruera atribuyó el "deficiente estado de la educación en todo el país" a una "excesiva concentración del poder de decisión en la conducción educativa del gobierno central." Para graficar ese postulado, caracterizó al ministerio de Educación de la nación de monstruoso, el cual según sus palabras, agotaba "gran parte de los recursos para la enseñanza de toda la república" y también limitaba sus posibilidades". ${ }^{9}$ Ese discurso fue el argumento de una resolución dictada poco después del encuentro para acelerar el proceso de descentralización (GUDELEVICIUS, 2009, p. 9).

A mediados del mes de septiembre volvió a reunirse el CFE en el marco

\footnotetext{
${ }^{6}$ Ministerio de Cultura y Educación. CFE. III Asamblea Extraordinaria, Buenos Aires, 10 de mayo de 1976, Centro Nacional de Documentación e Información Educativa (Cenide), Buenos Aires.

${ }^{7}$ El Diario, 11/5/76, p. 1.

${ }^{8}$ El Diario, 12/5/76, p. 2.

${ }^{9}$ El Diario, $13 / 5 / 76$, p. 3 .
} 
de la V Asamblea Ordinaria que se realizó en Tucumán, gobernada por el general Antonio Bussi. La Asamblea trató y aprobó un plan de "coordinación de acciones entre la nación y las provincias" que constaba de dos etapas. Durante la primera, el financiamiento quedaría en manos de la nación y durante la segunda recaería en estas últimas. ${ }^{10}$

Cuando el 30 de noviembre se reunió la Comisión de Pautas Presupuestarias para tratar el apoyo financiero de la Nación a las provincias, solo asistieron y enviaron trabajos representantes de las regiones Sur y NOA. Ante el temor fundado de que los recursos previstos para atender las escuelas transferidas fueran mayores a los que reconocía la nación, las dos regiones que participaron solicitaron un relevamiento catastral de la infraestructura edilicia y del personal, teniendo en cuenta de los aspectos salariales y previsionales. Con ello se pretendía fijar una política de apoyo financiero que debía actualizarse en forma permanente. Por su parte, la nación propuso la intervención de representantes del Ministerio de Economía.

La Comisión sugirió que la Secretaria Técnica del CFE convocara a reuniones regionales para tratar el tema durante el mes de diciembre. ${ }^{11}$ De éstas debía surgir un documento regional que contemplara un cronograma tentativo para la transferencia de funciones, el apoyo financiero de la Nación que se estimaba imprescindible para esas etapas y las sugerencias sobre las bases financieras y legales que sería necesario implementar para el traspaso total de servicios. Con estos documentos regionales se realizó una reunión plenaria en marzo de 1977 para producir un documento base. ${ }^{12}$

El documento base fue tratado y aprobado en abril de 1977 en la VI

\footnotetext{
10 Ministerio de Cultura y Educación. CFE. V Asamblea Ordinaria del CFE. Tucumán, 17 de septiembre de 1976, Cenide, Buenos Aires y El Diario, 18/9/76, p. 3.

${ }^{11}$ Consejo Federal de Educación. Comisiones Permanentes. En Escuelas Nacionales transferidas a provincias. Nivel primario. Antecedentes. Archivo del Consejo General de Educación. Entre Ríos.

${ }^{12}$ Clarín, 5/3/77, p. 9.
} 
Asamblea Ordinaria del CFE realizada en Posadas. ${ }^{13}$ Consignaba y caracterizaba las acciones necesarias para transferir los establecimientos educativos y encomendaba al Comité Ejecutivo que elaborara las pautas para una futura ley. Estimaba la concreción en los primeros meses de 1978, previamente a lo cual era necesario actualizar los estatutos docentes para que tuvieran criterios básicos, fijar los contenidos mínimos, la validez efectiva de los títulos y la participación de un régimen flexible de equivalencias. Asimismo, los coordinadores regionales consideraron imprescindible intensificar la preparación psicológica, en primer término, de la comunidad educativa, y en segundo término de la población en general. ${ }^{14}$

Una editorial de El Diario, publicada el día que iniciaba la Asamblea, enumeraba las dificultades que conllevaría el traspaso, entre las que se encontraban el régimen previsional del personal docente, administrativo y auxiliar, las condiciones de los edificios escolares y de su equipamiento, los recaudos institucionales y la adecuación a la legislación, el nivel de aptitud y los mecanismos financieros de las diferentes administraciones para recibir los servicios transferidos y atender a su crecimiento, la adecuación de los aspectos técnicos docentes a la nueva situación, la aplicación de los contenidos mínimos, la racionalización y redimensionamiento de un sistema educativo unificado y en etapas inmediatas para cumplir el objetivo propuesto. ${ }^{15}$

Como hemos visto, el argumento de la gestión de Bruera residía en equipar centralización, burocratización e ineficiencia, en contraste a descentralización, autonomía y eficiencia. Si bien se presentaron una serie de motivos "pedagógicos" no se hizo un gran esfuerzo por ocultar el trasfondo fiscal de la medida. Así, en la Asamblea de mayo, señalaba que el ministerio de Educación agotaba gran parte de los recursos destinados a la educación para todo el país y en la de septiembre, expresaba que la descentralización debía incluir el

\footnotetext{
13 Ministerio de Cultura y Educación. CFE. VI Asamblea Ordinaria, Posadas, 30 y 31 de marzo y 1 de abril de 1977, Cenide, Buenos Aires y El Diario, 30/3/77.

${ }_{14}$ El Diario, 30/3/77, p.6 y El Diario, 3/4/77, p. 5.

${ }_{15}$ El Diario, 30/3/77, p. 6.
} 
aspecto económico que había fracasado en los intentos previos. ${ }^{16}$ De hecho, en marzo de 1977, advertía que tenía entre sus propósitos "ofrecer soluciones a múltiples problemas económicos". ${ }^{17}$

Las objeciones de los gobiernos provinciales, estuvieron presentes desde un primer momento y llevaron a la necesidad de apelar a distintos mecanismos de legitimación. Como señala Rodríguez (2011, p. 36), al incluir la participación de todos los ministros educativos de la Argentina a través del CFE, el gobierno dictatorial quiso demostrar a la opinión pública que las decisiones eran producto de un consenso aunque, en la práctica, se tomaban desde el ministerio y el Poder Ejecutivo Nacional.

Junto con el CFE, otro mecanismo de búsqueda de legitimidad consistió en la apelación a los principios federales de la Constitución nacional. En mayo de 1976 el ministro Bruera señaló que la transferencia surgía "de claros preceptos constitucionales, que en nada invalidan los principios de las autonomías provinciales". ${ }^{18} \mathrm{Al}$ introducir la reunión que se llevó a cabo en Posadas en abril de 1977, reiteraba que los traspasos se efectuarían en base a "los dispositivos vigentes de la Constitución Nacional y de las constituciones provinciales" y que por ello mismo no era factible transferir los establecimientos medios. ${ }^{19}$ Sin embargo, las provincias mantuvieron su rechazo a la medida.

Bruera, que a comienzos de marzo había desmentido su renuncia, la presentó el 21 de abril, aunque continuó en el cargo hasta fines de mayo cuando fue reemplazado por el ministro del interior -general Albano Harguindeguyquien se mantuvo hasta mediados de junio cuando asumió el tucumano Juan Catalán. Su alejamiento, el primero del gabinete nacional, se debió a que había reclamado un mayor presupuesto para el área, pero también a la falta de acuerdo

\footnotetext{
${ }_{16}$ Ver El Diario, 13/5/76 y El Diario, 12/9/76. Tampoco lo negaba al referirse a la subvención a las escuelas privadas: "esto significa un gran ahorro, pues se eliminan gastos que tendrían que efectivizarse si estos establecimientos estuvieran a cargo del Estado.” El Diario, 16/11/76, p. 1.

${ }_{17}$ El Diario, 30/3/77, p. 6.

${ }_{18}$ Clarín, 6/5/76, p. 14.

19 El Diario, 30/3/77, p. 2.
} 
respecto a la implementación de la transferencia (RODRÍGUEZ, 2015).

\section{LA GESTIÓN DE CATALÁN: EL TRASFONDO FISCAL DE LA MEDIDA Y LA OPOSICIÓN DE LAS PROVINCIAS}

Catalán afirmaba que la transferencia no tenía "connotaciones económicas ni razones presupuestarias. Suponerlo sería considerar que el Gobierno Nacional desperdicia a la educación pública argentina”. ${ }^{20}$ Sin embargo, a contramano de lo expresado y de las acciones consensuadas con Bruera, la política relativa a las transferencias tomó otro rumbo. El ministro de Economía Martínez de Hoz alertó a los ministros provinciales que debían atender con recursos propios los costos totales que demandaran las transferencias, incluyéndolas para elaborar los presupuestos de 1978. Esos recursos podían provenir de restricciones en las erogaciones totales o de mayores esfuerzos en la administración tributaria local. De esta forma, se abandonaba la posibilidad de que en una primera etapa las provincias no tuvieran que financiar las escuelas nacionales con sus recursos.

El 5 de septiembre de 1977, los representantes del NEA se reunieron en Corrientes. Allí se señaló que la alternativa presentada por Martínez de Hoz era "totalmente desfavorable" para las provincias. Se argumentó que los esfuerzos solicitados eran superiores a los realizados por la nación y que la medida no tenía otra finalidad "que la de buscar mejorar la posición financiera de la administración pública nacional a costa de nuevos sacrificios de las provincias”. Asimismo se acusaba a la nación de no dar a conocer la metodología con la que se había calculado el costo para la atención de los servicios transferidos y de estimar valores muy por debajo de los entonces actuales, a lo cual se sumaban las

${ }_{20}$ El Diario, 22/11/77, p. 17. 
deficiencias en la infraestructura de los edificios. ${ }^{21}$

Los representantes del NEA recomendaron que las transferencias fueran acompañadas por los recursos nacionales que aseguraran el ejercicio 1978 y los posteriores "teniendo en cuenta cubrir la planta funcional necesaria para una adecuada prestación de los servicios”. En ese presupuesto además de las previsiones de 1977 se debían agregar los recursos para dar lugar a las mejoras salariales acordadas y a la incidencia por crecimiento vegetativo. Las previsiones debían efectuarse teniendo en cuenta la esperada variación del nivel de precios para todo el año y el encarecimiento en la locación de inmuebles. Otra de las demandas, como muchas escuelas funcionaban en edificios que necesitaban urgente reparación, era que las obras en ejecución tuvieran la financiación necesaria para la optimización gradual de la infraestructura edilicia. Asimismo, se solicitaba que en caso de que los recursos de la coparticipación resultaran insuficientes el déficit fuera financiado "mediante aportes no reintegrables del tesoro nacional". ${ }^{22}$

A fines de octubre el comité ejecutivo del CFE trató el tema de las transferencias y convocó a los ministros de educación a una Asamblea Extraordinaria que se realizaría en la segunda quincena de noviembre en la ciudad de Mendoza. Poco después Catalán anunció que el proyecto de ley estaba siendo estudiado por el Poder Ejecutivo, que sería aprobado en brevedad y concretado ese mismo año. Expresó que, una vez transferidas "de ser ello necesario" el gobierno nacional continuaría pagando al personal por el término de tres meses, pero que "los montos desembolsados deberían ser luego devueltos". Tanto el ministro de Educación como el de Economía, sostenían que las provincias estaban "en condiciones presupuestarias de recibir las escuelas que la Nación les traspasara, dado que tendrán una mejor recaudación fiscal”. ${ }^{23}$

\footnotetext{
${ }^{21}$ Ministerio de Educación y Cultura. Corrientes. Reunión del NEA, septiembre de 1977. En Escuelas Nacionales transferidas a provincias. Nivel primario. Antecedentes. Archivo del Consejo General de Educación. Entre Ríos.

${ }^{22}$ Ministerio de Educación y Cultura. Corrientes. Reunión del NEA, op. cit.

23 Clarín, 27/10/77, p. 18.
} 
Los reclamos que expresaron el desacuerdo con la medida se acrecentaron ante su inminencia. Así, el ministro de Cultura y Educación de Mendoza, aunque señalaba que la transferencia era necesaria e impostergable, advertía la insuficiencia del presupuesto que agravaba los inconvenientes de infraestructura existentes. ${ }^{24}$ También la prensa difundía los pronunciamientos del jefe de la cartera de Bienestar Social de Córdoba. Al igual que en el caso de Mendoza, si bien expresaba que acataría "la decisión adoptada por la nación”, el funcionario no dejaba de advertir que se carecía de información acerca de los recursos que se necesitaban para absorber los establecimientos y que en la $\mathrm{V}$ Asamblea Ordinaria de 1976 la posición de los funcionarios cordobeses se orientó a solicitar que el traspaso comprendiera solamente los aspectos operativos. Además, subrayaba que había varias escuelas-rancho y algunos edificios "en estado francamente deplorable". ${ }^{25}$ Del mismo modo, representantes de Jujuy manifestaron las dificultades presupuestarias y si bien señalaron las ventajas administrativas de la descentralización, se realizaron sugerencias respecto al financiamiento. ${ }^{26}$

Desoyendo los reclamos, Catalán afirmaba que "oficialmente no tienen noticias de la disconformidad de ningún gobierno provincial.” ${ }^{27}$ Asimismo, el secretario de Educación, Perramón Pearson, justificaba la transferencia diciendo que "todo lo que puede hacer el municipio no tiene por qué hacerlo la provincia; lo que pueden hacer las provincias no tiene por qué hacerlo la Nación y a su vez no se justifica que haga el Estado lo que están en condiciones de hacer los particulares." 28 Para ello se apoyaron en las declaraciones de los representantes de algunos gobiernos que manifestaron "estar preparados" para la transferencia, como son los casos de Formosa y Entre Ríos. ${ }^{29}$

\footnotetext{
${ }_{24}$ Clarín, 2/11/77, p. 17.

25 Clarín, 4/11/77, p. 16.

${ }^{26}$ Clarín, 7/11/77, p. 17.

${ }_{27}$ El Diario, 5/11/77, p. 3.

${ }^{28}$ Clarín, 8/11/77, p. 17.

${ }_{29}$ Clarín, 10/11/77, p. 19 y Clarín 12/11/77, p. 17.
} 
En la Primera Reunión de Ministros de Educación realizada en 1966 las autoridades de Entre Ríos habían manifestado que no estimaban factible la transferencia "si la nación no provee en forma permanente y actualizada anualmente, la totalidad de los recursos que destinaba al mantenimiento de las escuelas de su dependencia”. $3^{\circ}$ Por otra parte, la provincia no firmó ningún convenio a lo largo de esa década. Sin embargo, a fines de 1977, el interventor de facto Rubén Di Bello sostenía que "podría absorber sin mayores problemas las escuelas nacionales que serán transferidas este año”. Señalaba que el costo del sistema nacional educativo no superaría el 4 o $5 \%$ del presupuesto provincial aunque reconocía que "el cambio tendría proporciones considerables, si se piensa que el sistema nacional constituye un $20 \%$, aproximadamente, del total que rige el gobierno escolar provincial" y podía "frenar el plan de obras". ${ }^{31}$ De todas formas, la situación de Entre Ríos era menos aguda que la de otras provincias. En una nota posterior la prensa hablaba de un $25 \%$ del presupuesto educativo, que representaba un porcentaje mucho menor que, por ejemplo, el de Corrientes $(176 \%) .3^{2}$

El 3 de noviembre de 1977, en el marco de un encuentro regional del NEA, se aprobó un documento elaborado por la provincia de Entre Ríos titulado "Coordinación inmediata y efectiva entre jurisdicción nacional y provincial". Allí se presentaron acciones tanto de la nación como de la provincia para el periodo inmediatamente posterior a la ley de transferencia, que debían estar proyectadas y preparadas para ser puestas en ejecución. 33

Los días 20 y 21 de noviembre de 1977 se llevó a cabo en Mendoza la V

\footnotetext{
30 Argentina. Secretaría de Estado de Cultura y Educación. Reunión de Ministros de Educación. Informe Final. Cenide, Buenos Aires, 1966, p. 64. Asimismo en la reunión de 1968 las autoridades de la provincia afirmaban que la transferencia debía realizarse "en base al traspaso total de los recursos mediante modificación del índice de co-participación.” Ver Argentina. Secretaría de Estado de Cultura y Educación. Tercera Reunión de Ministros de Educación. Informe Final. Cenide, Buenos Aires, 1968, p. 205.

${ }^{31}$ Clarín, 10/11/77, p. 19.

${ }^{32}$ Clarín, 23/11/77, p. 16.

33 Entre Ríos. Consejo General de Educación. Estructura legislativa necesaria para la transferencia de los servicios. Paraná, 3 de noviembre de 1977.
} 
Asamblea Extraordinaria del CFE donde se recomendó el cumplimiento de la transferencia "conforme con la política enunciada por el gobierno nacional". Catalán insistía en que debía producirse antes de antes de que terminara ese año o a más tardar al comenzar el periodo lectivo 1978, con el consentimiento de los gobernadores y ministros de educación.34 En dicha Asamblea se trató el anteproyecto de ley, según el cual, se haría en una etapa y sin apoyo financiero de la nación, y el decreto reglamentario que fijaba un plazo breve para que la nación continuara liquidando y abonando las remuneraciones del personal transferido.

El anteproyecto fue objetado por todos los ministros de Educación que cuestionaron el aspecto presupuestario, la incompatibilidad jurídica entre las legislaciones sobre obras sociales y regímenes previsionales y la coordinación de servicios educativos. En un documento elaborado durante una reunión reservada expresaban que: "la nación, en los casos en que se provoque un desequilibrio en los presupuestos provinciales [...] debe acudir necesariamente en auxilio de las finanzas provinciales”. 35

Las tensiones descriptas conllevaron que a comienzos de 1978 quedara trunca la promesa del ministro Catalán de transferir las escuelas previamente al inicio del ciclo lectivo. Entre los argumentos para explicar las demoras, omitía hablar de la oposición de los diferentes gobiernos subnacionales y refería al "trámite burocrático" y los ministerios involucrados: Interior, Economía y Planeamiento. Sin embargo, la prensa dejaba ver que más allá de ese motivo, el retraso podía estar vinculado con "las severas críticas que, respecto a ciertos aspectos de la misma, hicieron algunos sectores". ${ }^{36}$

A mediados de febrero, El Diario publicaba la respuesta del ministro quien había afirmado que "es una decisión política de la Junta Militar" y que "el 13 de marzo con o sin ley, la Nación tomará las providencias necesarias para que

\footnotetext{
${ }^{34}$ Ministerio de Cultura y Educación. CFE. V Asamblea Extraordinaria. Mendoza, 20 y 21 de noviembre de 1977, Cenide, Buenos Aires y Clarín, 22/11/77, p. 17.

${ }^{35}$ Ministerio de Cultura y Educación. CFE. V Asamblea Extraordinaria. Mendoza, 20 y 21 de noviembre de 1977, Cenide, Buenos Aires y Clarín, 23/11/77, p. 21.

${ }^{36}$ El Diario, 2/2/78, p. 3.
} 
las provincias encaren su responsabilidad con respecto a educación primaria y se pueda iniciar el proceso de las transferencias". 37 No obstante, las clases comenzaron sin que el proyecto de ley hubiera ingresado a la Comisión Asesora Legislativa.

El 10 de marzo se reunió en la ciudad Paraná la Comisión de Coordinación de Acciones de la Región del NEA. Se designó como presidente a la representación de Formosa y como secretario a la de Entre Ríos, las dos provincias que habían manifestado la viabilidad de la medida y las que tenían la menor cantidad de escuelas nacionales de la región. En dicha reunión se aprobó el anteproyecto de Entre Ríos para ser tomado como propuesta a ser presentada a la nación con el propósito de adoptar criterios comunes. El anteproyecto se aprobó con la aclaración de que, en el proceso de la transferencia, cada provincia limitaría las distintas acciones y la coordinación de ellas a las necesidades y características propias. 38

Al igual que su antecesor, Catalán buscó legitimidad en la Constitución. Afirmó que la transferencia era "simplemente restituir las competencias constitucionales históricas del país, las provincias tienen no solo la obligación, sino el derecho y la responsabilidad constitucional de impartir la instrucción primaria en su territorio". 39 Incluso, los docentes de Capital Federal utilizaban esos términos para cuestionar la transferencia a la municipalidad de la ciudad de Buenos Aires: "si malo puede ser, en cuanto a resultados prácticos, el traspaso de escuelas a provincias carentes de medios para sostenerlas, podría explicarse con disposiciones constitucionales”. ${ }^{40}$

De hecho, fue el argumento constitucional el que requirió que la situación de las escuelas de Capital Federal y de Tierra del Fuego se contemplara por una ley diferente. En este sentido, Catalán insistió que en que "la municipalidad de

\footnotetext{
37 El Diario, 13/2/78, p. 1.

${ }^{38}$ Gobierno de Entre Ríos. Región del Nordeste Argentino. Comisión de Coordinación de Acciones. Reunión del 10 de marzo de 1978.

39 El Diario, 4/11/77, p. 3.

40 El Diario, 6/11/77, p. 4.
} 
Buenos Aires recibirá las escuelas solamente por delegación ya que no es un organismo de la constitución sino una creación legal". ${ }^{4}$ Ello implicó también que debieran retroceder en las intenciones de traspasar el nivel medio. ${ }^{22}$ Tomando el análisis efectuado respecto al artículo 5 de la Constitución de 1853, el ministro advertía que "una política de transferencia de los establecimientos de nivel medio, en principio no resultaba factible". 43 Así, fue dejada de lado la pretensión que el gobierno nacional había manifestado en la I Asamblea del CFE en 1972 y que se había mantenido presente durante 1976.44

En lo que respecta al CNE, Catalán aseguraba su subsistencia, afirmando que se quedaría a cargo de las Escuelas de Aplicación "para hacer en ellas las experiencias pedagógicas que considere conveniente".45 A mediados de noviembre informó que remitiría junto a los dos proyectos de transferencia, otro que establecería las bases por las cuales el CNE dictaría la política para la educación preprimaria y primaria de todo el país. ${ }^{46}$ Sin embargo, dicho proyecto no tuvo continuidad y la información presentada por el Ministerio de Educación fue escasa y confusa, lo cual da cuenta de la intención de retrasar su supresión para no sumar más conflictos. 47 Finalmente, en 1980 un año antes del centenario de su creación, se sancionó la Ley 22.221 que reemplazó al CNE por una Dirección Nacional de Educación Primaria.

Otro argumento expresado durante 1977 para legitimar la transferencia consistió en dar a conocer la posibilidad de un crédito de 100 millones de dólares, provenientes del Banco Interamericano de Desarrollo, que según el Ministerio de Cultura y Educación sería "para asistir a las provincias en el trance de recibir las

\footnotetext{
${ }^{41}$ Clarín, 8/11/77, p. 17.

${ }^{2}$ El Diario, 30/3/77, p. 3.

43 Ver Ministerio de Cultura y Educación. CFE. VI Asamblea Ordinaria. Posadas, 30 y 31 de marzo y 1 de abril de 1977, Cenide, Buenos Aires y El Diario, 30/3/77.

44 Fue en 1992, durante la presidencia de Carlos Menem, que se transfirieron el resto de los niveles educativos menos el universitario.

45 Clarín, 29/10/77, p. 12.

${ }^{46}$ Clarín, 18/11/77, p. 19.

47 Ver El Diario, 22/2/78, p. 3 y El Diario, 7/6/78, p. 1.
} 
escuelas primarias nacionales". Ante dicha ocasión el gobernador de Salta expresaba que "se contaría con esa asistencia para solventar el costo de los nuevos servicios educativos, que las provincias iban a estar obligadas a brindar”. 48

En abril 1978, cuando los proyectos de transferencia ingresaron a la Comisión Asesora Legislativa, la prensa volvió a hacer referencia al préstamo que había solicitado el gobierno nacional "para un programa de construcciones escolares para la eliminación de escuelas rancho" y que debía ser considerado antes de fin de año. Esta vez, en lugar de 100, se hablaba de 60 millones de dólares. ${ }^{49}$ Finalmente, en el mes de diciembre, el préstamo fue otorgado por un valor de 50 millones, con una contraparte similar del gobierno nacional y con una redefinición de los objetivos que excedían la cuestión edilicia, dando lugar al Programa de Expansión y Mejoramiento de la Educación Rural (EMER).

En un trabajo anterior (PETITTI, 2019) teniendo en cuenta, por un lado, las coincidencias entre la fechas del decreto que aprobó la transferencia y aquel que dio inicio al Programa, y por otro lado, los diferentes discursos que lo acompañaron, señalamos que el Programa EMER consistió en una instrumento que utilizó el estado nacional durante el gobierno de la dictadura militar a través del financiamiento externo, más que para contribuir a atenuar sus consecuencias económicas, como una forma de otorgar legitimidad a la medida.

\section{LA TRANSFERENCIA DE ESCUELAS EN LA PROVINCIA DE ENTRE RÍOS}

En el mes de abril de 1978, sin que se diera lugar a las correcciones introducidas, los dos proyectos de ley de transferencia (a la municipalidad de Buenos Aires y al territorio de Tierra del Fuego por un lado, y a las provincias por el otro), ingresaron a la Comisión Asesora Legislativa y fueron declarados de

\footnotetext{
${ }^{48}$ Clarín, 20/11/77, p. 16.

49 El Diario, 17/4/78, p. 1.
} 
trascendencia. De todas formas la Asamblea Nacional prevista a realizarse la segunda quincena de marzo de 1978 debió ser anulada por las demoras. $5^{\circ}$ En Entre Ríos, el gobernador afirmaba que tenía recursos para afrontar la transferencia. Sin embargo, no ocultaba la problemática administrativa y edilicia, especialmente, en lo relativo a las diferencias de puntaje en favor del docente nacional que generaría una desventaja para los locales en los concursos; las diversidades de los programas y los riesgos de desalojo ante la cantidad de inmuebles bajo el régimen locativo. ${ }^{51}$

Finalmente, a comienzos de junio se sancionaron las leyes 21.809/78 y 21.810/78 sin tener en cuenta las demandas de las jurisdicciones que "aceptaron" recibir las escuelas de la nación. El decreto reglamentario 1.231 sancionado el mismo día, dispuso que la Nación continuaría abonando las remuneraciones del personal y servicios "hasta que las provincias puedan atenderlos, con un plazo máximo de nueve meses" a partir del primero de enero de 1978.52 La prensa dejaba entrever las presiones que recibieron los diferentes gobiernos: "en general, dieron su conformidad después de diálogos mantenidos al respecto por el ministro del Interior, general Albano Harguindeguy con los gobernadores de provincias, a veces en reuniones generales, o, por separado si así lo aconsejaban las circunstancias". .53

Después de la transferencia, la prensa publicó una editorial que con tono crítico refería a la situación. Señalaba que el traspaso:

\footnotetext{
Está más dirigido hacia la materialidad escolar y su funcionalidad, que hacia la política educativa [...] siendo la orientación nacional, como lo fue siempre, la expresión de deseos de los ministros firmantes del considerando solo queda reducida a una frase más [...] Es muy probable que surjan inconvenientes de índole económica, también de alcances de

50 El 12 de mayo el comité ejecutivo del CFE se reunió en Buenos Aires para elaborar la agenda a tratar en la próxima asamblea, pero no hubo consenso acerca de la fecha. El Diario, 13/5/78, p. 3 .

${ }^{51}$ El Diario, $12 / 4 / 78$, p. 3.

$5^{2}$ Poder Ejecutivo Nacional. Decreto 1.231/78, 5 de junio de 1978.

53 El Diario, 7/6/78, p. 1.
} 
las respectivas jurisdicciones y seguramente de fricciones por los nuevos mandatos docentes. También será muy difícil que una misma tendencia educativa sea aplicada con parecida ejecutoriedad por todas las provincias. 54

Además, El Diario, daba cuenta de las voces disonantes que sostenían que "si en breve la Nación no crea un fondo escolar de ayuda financiera a las provincias, no será posible dar respuesta al crecimiento vegetativo del sistema educativo y en cambio, se correrá el riesgo de la atomización del mismo”.55 El secretario de Educación, debió salir a responder que "el gobierno nacional resguardará la unidad del sistema educativo y el nivel de enseñanza, una vez realizado el traspaso". ${ }^{56}$

A partir de la sanción de la ley 21.809/78, el Ministerio de Cultura y Educación comenzó a firmar los convenios para dar forma jurídica al hecho. Así, como decíamos en la introducción, en 1978 se transfirieron masiva y obligatoriamente alrededor de 6.500 establecimientos, supervisiones y juntas de clasificación, 70.000 docentes y 900.000 alumnos. Según el gobierno nacional ello demandarían una erogación de 250.000 millones de pesos. 57 El compromiso recayó de manera desigual según las realidades socioeconómicas y la cantidad de las escuelas nacionales que variaba enormemente según los casos. En San Juan por ejemplo eran 121, en Neuquén 153, pero otras provincias poseían más de 500, como son los casos de Santa Fe, Chaco, Córdoba o Corrientes. ${ }^{8}$ La que más recibió fue Santiago del Estero - 717 -, elegida como sede del “Día T” para dar carácter simbólico a los mencionados traslados del sistema educativo”.59

La jornada se llevó a cabo el 17 de julio. Según el texto de la declaración,

\footnotetext{
54 El Diario, 9/6/78, p. 4.

55 El Diario, $18 / 6 / 78$, p. 3

${ }^{6}$ El Diario, 23/6/78, p. 3

57 El Diario, 20/7/78, p. 3.

${ }^{8}$ Argentina. Secretaría de Estado de Cultura y Educación. Tercera Reunión de Ministros de Educación. Informe Final. Cenide, Buenos Aires, 1968.

${ }_{59}$ El Diario, 7/6/78, p. 1.
} 
la ceremonia revestiría "un acto de reivindicación del federalismo." Se enunciaba que las causas que determinaron la creación de escuelas en virtud de la Ley 4.874 y en los ex Territorios Nacionales habían perdido vigencia y que la descentralización significaba una acción educadora más real y efectiva al reconocer las diferencias regionales, las necesidades del medio e integrarse en el CFE. ${ }^{60}$ El 18, también en Santiago del Estero se concretó la VII Asamblea del CFE. Al mes siguiente, el presidente de facto Videla le pidió la renuncia a Catalán. ${ }^{61}$ Nuevamente asumió el ministro del interior Albano Harguindeguy, quien estuvo en la cartera hasta el mes de noviembre cuando fue reemplazado por Juan Rafael Llerena Amadeo.

Desde octubre de 1978 las provincias, la municipalidad de Buenos Aires y Tierra del Fuego debieron pagar todos los gastos que demandaban las escuelas nacionales, lo que obligó a los estados a elevar en forma considerable las partidas destinadas a educación. ${ }^{62}$ Además, las provincias debieron adecuarse legalmente a la incorporación de las nuevas escuelas. La Comisión Permanente de Coordinación de Acciones -a partir de un cuestionario remitido a todas las jurisdicciones- realizó una evaluación de la implementación de la transferencia. La cual, a pesar de la escasa fiabilidad de la información, da cuenta de las diferentes acciones que debieron encarar las provincias para responder a las dificultades administrativas y fiscales. ${ }^{63}$

El CGE de Entre Ríos, creó una Comisión Técnica en la Secretaría de Estado de Cultura y Educación cuyo objetivo era la planificación y organización de las medidas legales y administrativas para efectivizarla. La comisión estuvo a cargo de un coordinador, un asesor legal, una secretaria técnica y un técnico

\footnotetext{
${ }^{60}$ Declaración de Santiago del Estero. En Dirección Nacional de Investigación, experimentación y perfeccionamiento educativo. Transferencia de los servicios educativos dependientes del Consejo Nacional de Educación. Cenide, Buenos Aires, 1980. p. 8.

${ }^{61}$ El Diario, 29/8/78, p. 3.

${ }^{62}$ El Diario, $18 / 10 / 78$, p. 3.

63 Los aspectos estudiados fueron: organización administrativa, personal, infraestructura y equipamiento, aspectos técnicos-docentes, previsión, fondos e información sobre las acciones para favorecer la integración. Ver Dirección Nacional de Investigación. op. cit.
} 
Docente de Planeamiento que eran afectados de sus tareas y que conocían los antecedentes. 64

$\mathrm{El} 7 \mathrm{de}$ julio, antes de que se firmara el convenio en Santiago del Estero, el gobierno de Entre Ríos sancionó la Ley 6.175/78 por la que aceptaba todas escuelas primarias, Supervisiones y Juntas de clasificación dependientes del CNE y radicadas en Entre Ríos. Durante ese mes el CGE dictó dos nuevas resoluciones. La primera, tenía como finalidad garantizar durante el periodo de transición que se mantuviera la estructura de los organismos y establecimientos transferidos pero sin obviar su dependencia del CGE. Para ello instituyó que el supervisor seccional y el personal de su jurisdicción pasaran a depender de dicho organismo. ${ }^{65}$ La segunda, teniendo en cuenta que el curriculum vigente era diferente en ambas instituciones, dejaba sentado que mantendría en las escuelas nacionales el currículo, los sistemas de evaluación, promoción, planificación y las técnicas de enseñanza-aprendizaje que se venían aplicando hasta la fecha. Disponía que Dirección de Enseñanza adoptara las medidas necesarias para que el año siguiente los contenidos fueran homogéneos y se implantara un plan de perfeccionamiento docente y actualización. Se pretendía que a partir del próximo periodo lectivo se encontraran en condiciones de aplicar nuevos curriculum, técnicas de enseñanza aprendizaje, sistemas de evaluación, promoción y planificación. ${ }^{66}$

La Supervisión Seccional de Escuelas de Entre Ríos contaba con un Secretario Técnico, nueve Supervisiones de zona y 234 escuelas con 1583 cargos docentes. La distribución por departamento -que en ese entonces eran 15reflejaba la heterogeneidad existente a nivel nacional. De las 234, cinco departamentos tenían menos de diez: Feliciano 4 (de un total de 31), Gualeguay 6 (de 66), Federación 7 (de 69), Diamante 7 (de 50) y Victoria 9 (de 52). Por otra parte La Paz contaba con 12 (de 98), Tala 13 (de 49), Nogoyá 15 (de 95), Concordia

\footnotetext{
64 Resolución CGE Entre Ríos 1264, 27/6/78.

65 Resolución del CGE número 689 con fecha 26/7/78 art 1.

${ }^{66}$ Resolución del CGE número 690 con fecha 26/7/78.
} 
15 (de 78) y Federal 16 (de 56). Mientras que otros cinco departamentos poseían más de 20: Colón 26 (de 78), Gualeguaychú 23 (de 137), Uruguay 27 (de 106), Villaguay 27 (de 107) y Paraná 27 (de 175). ${ }^{67}$

Con el fin de encuadrar su distribución, se dispuso la transformación del cargo de Secretario Técnico en su equivalente de Superior Escolar de zona y la incorporación de las supervisiones y los establecimientos a las jurisdicciones de las direcciones departamentales. Los supervisores zonales transferidos quedaron bajo la jurisdicción del director Departamental de escuelas mientras durara su función en el departamento. La designación de personal docente interino o suplente se continuaría realizando hasta finalizar el año en la forma prevista en el orden nacional, previa aprobación del director departamental.

El 16 de agosto, la Junta de Clasificaciones del CNE pasó a depender del CGE y se autorizó la inscripción para internos y suplentes al personal transferido, dando como plazo hasta el 30 de septiembre de ese año. ${ }^{68}$ Asimismo la División de Estadística y Censo debió asignar a las escuelas incorporadas nueva numeración con el siguiente al último que tenían las escuelas del Departamento donde estaban ubicadas. De este modo, se dejó sin efecto la identificación del orden nacional y se impusieron nuevos números para evitar la superposición. ${ }^{69} \mathrm{~A}$ fines de agosto, quedaron incorporados en el sistema provincial los docentes que hasta ese momento habían pertenecido al orden nacional. Ese mismo mes, la presidenta del CGE Alida Leonor Palomeque renunció y fue designada como interventora otra mujer, la Profesora María Olga del Azar de Guisto. ${ }^{70} \mathrm{Al}$ igual que en el ámbito nacional, la finalización de la transferencia coincidió con un cambio en la gestión educativa, vinculado además a la sucesión de dos interventores entre agosto y octubre de 1978.

El 12 de octubre se aprobó la resolución por la que se redistribuían las

\footnotetext{
${ }^{67}$ Resolución del CGE número 737 con fecha 9/8/78.

68 Resoluciones del CGE número 754 y 755 con fecha 16/8/78.

69 Resolución del CGE número 770 con fecha 23/8/78.

70 Poder Ejecutivo de la provincia de Entre Ríos. Decreto 33/78 Ministerio de Gobierno, Justicia y Educación.
} 
escuelas transferidas en nuevas zonas de supervisión escolar. En diez departamentos el incremento de establecimientos requirió aumentar una zona de supervisión, cuyos cargos se cubrieron con los de la nación que fueron adjudicados por orden de méritos a los ex supervisores nacionales con carácter de interino. También en octubre, se otorgó tiempo hasta fin de año para regularizar su situación a los docentes que se encontraban en uso de traslado transitorio en otros establecimientos del mismo territorio. Sin embargo, ante la demora ocasionada por la necesidad de cumplir los requisitos de distancia y antigüedad, se debió extender el plazo para la reubicación del personal durante todo el año 1979. ${ }^{71}$ Asimismo, en diciembre se dispuso que se extendiera a los ex docentes nacionales la misma hoja anual de concepto profesional que a los provinciales. 72

Anteriormente referíamos a una resolución del CGE que absorbía 234 establecimientos. Ahora bien, una normativa emitida en el mes de octubre, especificaba que se habían transferido 244 escuelas comunes, entre las cuales había 6 albergues, 26 de jornada completa y 212 de jornada simple. De esas últimas, solo 204 figuraban en el listado y se dividían en cuatro categorías: primera (34), segunda (23), tercera (100) y Personal Único (47). De las 26 de jornadas completa: 15 eran de primera categoría, 7 de segunda, 3 de tercera y una de personal único. De las 6 escuelas albergue 3 eran de segunda categoría y las restantes de tercera.73 Esto constituye un ejemplo de las variaciones en las cifras que ha sido advertida por Rodríguez a nivel nacional (2008b). Si revisamos las actas de inspección de la Entre Ríos, hacia 1964 se hablaba de 244 escuelas, de las cuales funcionaban 240.74 En 1968 documentos del CNE daban la cifra de 253. En 1977 la prensa nacional señalaba que funcionaban 236, con una población de 27.334 alumnos y 1.667 docentes. Del total citado, 169 funcionan en locales

\footnotetext{
${ }^{71}$ Resolución del CGE número 1718 con fecha 29/12/78.

${ }^{72}$ Resolución del CGE número 1181 con fecha 4/12/78.

73 Resolución del CGE número 341 con fecha 12/10/78.

74 Actas de Inspección de Escuelas Nacionales, 1932 hasta 1974. Paraná, 6/8/64, foja 115.
} 
propios, 34 en ámbitos alquilados y 33 en lugares cedidos sin cargo.75

Según un informe del Ministerio de Cultura y Educación publicado en 1980, solo seis provincias habían cerrado escuelas y Entre Ríos no estaba incluida. ${ }^{76}$ Sin embargo, si observamos en cada división administrativa la suma de las escuelas provinciales y las transferidas en 1978 y las comparamos con los establecimientos existentes en 1986, podemos advertir que en 12 de los 15 departamentos hubo una disminución del número de establecimientos que en toda la provincia alcanza la cifra de 83.77

\section{CONSIDERACIONES FINALES}

En este artículo pretendimos realizar una contribución en clave federal a los estudios que analizan la transferencia de establecimientos educativos primarios durante la dictadura. Concentrarnos en los dos años previos y los meses posteriores en la provincia de Entre Ríos y la región del NEA, nos permitió acercar la lente a problemas que se habían abordado en estudios realizados desde un marco nacional o que miraron la ciudad y la provincia de Buenos Aires. Tomando como base los aportes de esas investigaciones, hemos visto de qué manera, en el marco de la dictadura militar, fueron numerosas las voces que reclamaron por los problemas financieros en los que las provincias se verían involucradas para enfrentar el traspaso. Ante ellas, se llevaron a cabo una serie de mecanismos de legitimación que consistieron en la realización de reuniones de ministros en el marco del CFE, la apelación a la Constitución federal de 1853 y la toma de un crédito externo para financiar la edificación de escuelas y el perfeccionamiento docente en las zonas rurales, aquellas con mayor presencia de escuelas nacionales.

\footnotetext{
75 Clarín, 10/11/77, p. 19.

76 Dirección Nacional de Investigación... op. cit.

77 Resolución 341 del Consejo General de Educación de Entre Ríos, del 12/10/78 y Departamento Estadística y Censo Escolar. Consejo General de Educación. Marzo 1986.
} 
Asimismo pudimos advertir las diferencias entre las gestiones de los ministros de educación Bruera y Catalán en lo que respecta a las estrategias para llevar a cabo la transferencia y el grado de explicitación de los objetivos fiscales que subyacían a la medida. En este punto, al profundizar en el caso de Entre Ríos, hemos observado que la preponderancia que adquirió en la región del NEA durante la gestión de Catalán, evidencia la estrategia del gobierno dictatorial de apoyarse en determinadas provincias en el marco de encuentros regionales, con el fin de fragmentar lo reclamos y obtener legitimidad. Si bien en un primer momento Entre Ríos manifestaba no poder afrontar la transferencia si no contaba con los recursos, en 1977, fue tomada como ejemplo de aquellas provincias que podían enfrentarla.

Por otra parte, señalamos que las disparidades en las cifras respecto a las escuelas nacionales, daban cuenta del desconocimiento del costo financiero total que implicaba la transferencia. Este hecho, que era denunciado por los gobiernos provinciales, encubrió el impacto del cierre de establecimientos. Finalmente, nos interesó mostrar que además de los problemas fiscales que implicó el cierre de escuelas, la transferencia conllevó una serie de dificultades administrativas que no se terminaron de resolver en el transcurso del año.

Cabe dejar planteado el interrogante acerca de los resultados que arrojarían futuras investigaciones basadas en estudios comparados en ámbitos subnacionales, identificando los posicionamientos de los gobiernos provinciales al interior de las diferentes comisiones regionales, indagando las variaciones en el número de establecimientos transferidos, así como también analizando las estrategias fiscales y administrativas que las provincias debieron llevar a cabo para adecuarse a la incorporación de las escuelas nacionales.

\section{REFERENCIAS}

ALONSO BRÁ, Mariana. La descentralización a partir de las políticas de 
transferencia: el caso del sistema educativo argentino. Convergencia Revista de Ciencias Sociales, n. 18, p. 81-111, ene. 1999.

ASCOLANI, Adrián. Estrategias del Banco Mundial para el financiamiento de la educación en los países latinoamericanos. Educação, n. 31, v. 2, p. 139-156, 2008.

AYROLO, Valentina; VERDO, Genèviéve. Introducción al Dossier de difusión "Las provincias antes de la Nación en la Argentina. Entre la soberanía la autonomía y la independencia”, n. 75, p. 1-9, ene. 2016.

BOHOSLAVSKY, Ernesto; SOPRANO, Germán (ed.). Un Estado con rostro humano. Buenos Aires: Prometeo Libros, 2010.

BORDÓN, José Octavio. Historia y política de la descentralización y el gobierno educativo en la Argentina. In: OLIVEIRA ANDRADE, Dalilia (org.). Políticas educativas y territorios de articulación entre niveles de gobierno. Buenos Aires: Instituto Internacional de Planeamiento de la educación IIPEUnesco, 2010.

BRASLAVSKY, Cecilia. La discriminación educativa en Argentina. Buenos Aires: Miño y Dávila, 1985.

BRAVO, Cecilia. Los sentidos de la nación y el federalismo en la Argentina 1830-1880. Revista Historia y Memoria, n. 6, p. 205-232, 2013.

DE LUCA, Romina. La educación argentina en épocas de la última dictadura militar: regionalismo y descentralización del nivel primario de educación (19761983). Contextos Educativos, Universidad de La Rioja, n. 16, p. 73-88, 2003. Disponible en: https://ri.conicet.gov.ar/bitstream/handle/11336/5201/12911223-1-PB.pdf?sequence $=5$ \&isAllowed $=\mathrm{y}$

FALLETI, Tulia. Federalismo y descentralización en Argentina. Antecedentes históricos de la relación entre Nación y provincias y el escenario institucional de las nuevas relaciones intergubernamentales. In: Workshop Descentralización, Sociedad Civil y Gobernabilidad Democrática, Septiembre de 2001.

FILMUS, Daniel. La descentralización educativa en Argentina: elementos para el análisis de un proceso abierto. Revista Reforma y Democracia, CLAD, Caracas, p. 1-13, feb. 1998.

GARCÍA, Natalia. Historiografía y memorias en la educación argentina reciente: balances políticos-académicos y aportes desde un estudio de caso. 
Perspectivas e Diálogos: Revista de História Social e Práticas de Ensino, v. 1, n. 3, p. 173-198, ene./jun. 2019.

GUDELEVICIUS, Mariana. Educación primaria y proyecto político local. El caso de la MCBA durante la última dictadura argentina (1978-1982). In: JORNADAS INTERESCUELAS/DEPARTAMENTOS DE HISTORIA, Universidad Nacional de Cuyo, XIV, 2013, Mendoza. Disponible en: http://cdsa.aacademica.org/ooo010/1150.pdf

GUDELEVICIUS, Mariana. Un análisis de la política de transferencia de escuelas primarias durante la última dictadura argentina y el devenir de la situación laboral docente en ese proceso. 1976-1981. In: JORNADAS

INTERESCUELAS/DEPARTAMENTOS DE HISTORIA, Universidad Nacional del Comahue, XII, 2009, San Carlos de Bariloche. Disponible en: http://cdsa.aacademica.org/ooo-0o8/828.pdf.

KRAWCZYK, Nora. La reforma educativa en América Latina desde la perspectiva de los organismos multilaterales. Revista Mexicana de Investigación Educativa, v. 7, n. 16, p. 626-63, sept. 2002.

MORDUCHOWICZ, Alejandro. Descentralización educativa y capacidades institucionales de las Provincias. In: Aportes para el Estado y la administración gubernamental, n. 26, p. 39-55, 2009.

MORDUCHOWICZ, Alejandro. El federalismo fiscal-educativo argentino. In: OLIVEIRA ANDRADE, Dalilia (org.). Políticas educativas y territorios de articulación entre niveles de gobierno. Buenos Aires: Instituto Internacional de Planeamiento de la educación IIPE-Unesco, 2010.

ORLANSKY, Dora. Las políticas de descentralización. Desarrollo Económico, v. 38, n. 151, p. 797-896, oct./dic. 1998.

PETITTI, Mara. Los organismos financieros internacionales y la educación rural en Argentina. Un estudio del Programa EMER en la provincia de Entre Ríos (1978-1992). Avances del Cesor, 2019, en prensa.

RIVAS, Axel. Gobernar la educación: estudio comparado sobre el poder y la educación en las provincias argentinas. Buenos Aires: Ediciones Granica, 2004.

RODRÍGUEZ, Laura. La escuela intermedia revisitada: racionalización y revisión curricular en la provincia de Buenos Aires durante la última dictadura militar. Trabajos y Comunicaciones, Universidad Nacional de La Plata, Facultad de Humanidades y Ciencias de la Educación, v. 34, p. 35-61, 2008 a. Disponible en: 
http://www.memoria.fahce.unlp.edu.ar/library?a=d\&c=arti\&d=Jpr3723 .

RODRÍGUEZ, Laura. La primaria durante la dictadura: reforma curricular y "concentración" de escuelas rurales. Archivos de Ciencias de la Educación, Universidad Nacional de La Plata, Facultad de Humanidades y Ciencias de la Educación, v. 2, n. 2, p. 85-102, 2008b. Disponible en:

http://www.memoria.fahce.unlp.edu.ar/library? $a=d \& c=a r t i \& d=J p r 3177$.

RODRÍGUEZ, Laura. Las políticas educativas de las últimas dictaduras en el Cono Sur Latinoamericano. ¿Qué podemos comparar? Apuntes para la construcción de una agenda de investigación. In: ENCUENTRO

INTERNACIONAL DE ESTUDIOS COMPARADOS EN EDUCACIÓN, II, Buenos Aires, 18 al 20 de junio, 2009.

RODRÍGUEZ, Laura. Católicos, nacionalistas y políticas educativas en la última dictadura (1976-1983). Rosario: Prohistoria, 2011.

RODRÍGUEZ, Laura. Funcionarios y políticas educativas en Argentina (19761983). Cuadernos Chilenos de Historia de la Educación, Universidad Santiago de Chile, v. 2, n. 4, p. 62-85, jun. 2015.

RODRÍGUEZ, Lucía. La cuestión nación provincia en el desarrollo de la política educativa argentina. In: CONGRESO ARGENTINO DE ADMINISTRACIÓN PÚBLICA "ADMINISTRACIÓN PÚBLICA Y CUESTIÓN FEDERAL: LA RED NACIÓN, PROVINCIAS Y MUNICIPIOS”, V, San Juan, 2009.

RUIZ, Guillermo y MAUCERI, María. La finalización de la educación primaria nacional. Los últimos años de la acción directa del Estado nacional en la educación primaria. Período 1961-1978. Historia de la Educación, Anuario, Buenos Aires, v. 10, 2009.

TEDESCO, Juan, BRASLAVSKY, Cecilia; CARCIOFI, Ricardo. El proyecto educativo autoritario. Argentina (1976- 1982), Buenos Aires: Flacso, 1983.

EVA MARA PETITTI es profesora, magister y doctora en Historia por la Universidad Nacional de Mar del Plata. Investigadora adjunta del Consejo Nacional de Investigaciones Científicas y Técnicas (Conicet) y Jefa de Trabajos Prácticos en la Facultad de Ciencias Económicas de la Universidad Nacional de Entre Ríos. Desarrolla investigaciones en temas vinculados a la Historia Social de la Educación argentina durante el siglo XX. Es autora de 
Más allá de una escuela peronista. Políticas públicas y educación en la provincia de Buenos Aires (1946-1955).

E-mail: marapetitti@gmail.com

(b) http://orcid.org/0000-0001-8014-7181

Recebido em: 23 de dezembro de 2019

Aprovado em: 13 de junho de 2020

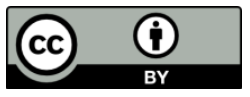

Revista História da Educação - RHE

Associação Sul-Rio-Grandense de Pesquisadores em História da Educação - Asphe

Artigo de acesso aberto distribuído nos termos de licença Creative Commons. 